\title{
PCSK9 mediates the oxidative low-density lipoprotein-induced pyroptosis of vascular endothelial cells via the UQCRC1/ROS pathway
}

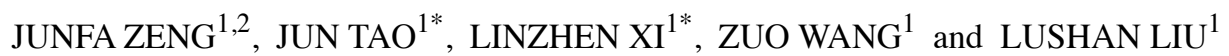 \\ ${ }^{1}$ Institute of Cardiovascular Disease, Key Laboratory for Arteriosclerology of Hunan Province, Hunan International Scientific \\ and Technological Cooperation Base of Arteriosclerotic Disease, Hengyang Medical College, University of South China; \\ ${ }^{2}$ The Second Affiliated Hospital of The University of South China, Hengyang, Hunan 421001, P.R. China
}

Received August 22, 2020; Accepted January 22, 2021

DOI: $10.3892 / \mathrm{ijmm} .2021 .4886$

\begin{abstract}
The present study aimed to explore the role and mechanisms of proprotein convertase subtilisin/kexin type 9 (PCSK9) in the oxidized low-density lipoprotein (oxLDL)-induced pyroptosis of vascular endothelial cells. For this purpose, human umbilical vein endothelial cells (HUVECs) were incubated with oxLDL $(100 \mu \mathrm{g} / \mathrm{ml})$ for $24 \mathrm{~h}$ to induce pyroptosis, which was detected using PI/hoechst 33342 double staining. The expression of pyroptosis-associated molecules was measured by western blot analysis and RT-qPCR Reactive oxygen species (ROS) and membrane potential were examined through ROS probe and JC-1 staining, respectively. PCSK9 and mitochondrial ubiquinol-cytochrome $c$ reductase core protein 1 (UQCRC1) protein were knocked down by small interfering RNA (siRNA). PCSK9 was overexpressed by lentivirus. The results revealed that oxLDL induced HUVEC injury, pyroptosis and inflammatory factor release, and upregulated the expression of PCSK9 protein in the HUVECs in a concentration-dependent manner. The silencing of PCSK9 expression with siRNA suppressed the oxLDL-induced damage to HUVECs, the release of inflammatory substances and the occurrence of pyroptosis. In addition, oxLDL inhibited UQCRC1 expression, promoted mitochondrial membrane potential collapse and damaged mitochondrial function; however,
\end{abstract}

Correspondence to: Professor Lushan Liu or Professor Zuo Wang, Institute of Cardiovascular Disease, Key Laboratory for Arteriosclerology of Hunan Province, Hunan International Scientific and Technological Cooperation Base of Arteriosclerotic Disease, Hengyang Medical College, University of South China, 28 W Changsheng Road, Hengyang, Hunan 421001, P.R. China

E-mail: liuls2000@163.com

E-mail: smt121101@163.com

*Contributed equally

Key words: atherosclerosis, oxidized low-density lipoprotein, proprotein convertase subtilisin/kexin type 9, oxidized low-density lipoprotein, pyroptosis, ubiquinol-cytochrome $c$ reductase core protein 1 , reactive oxygen species, endothelial cell these processes were reversed by the silencing of PCSK9. PCSK 9 overexpression induced the pyroptosis of HUVECs, the generation of ROS and the disorder of mitochondrial function by inhibiting UQCRC1. Therefore, PCSK9 mediates the oxLDL-induced pyroptosis of vascular endothelial cells via the UQCRC1/ROS pathway.

\section{Introduction}

Atherosclerosis (AS) is a chronic inflammatory arterial angiopathy and a common cause of cardiovascular diseases (1). The common pathological changes of AS include endothelial cell dysfunction, lipid deposition and vascular wall thickening (2). Vascular endothelial cells constitute the inner lining of arterial vessels, and their morphological integrity and functional homeostasis play an important role. Damaged integrity and dysfunction in vascular endothelial cells can initiate AS and thus lead to the development of severe clinical acute events, such as myocardial infarction and stroke (3). Proprotein convertase subtilisin/kexin type 9 (PCSK9) is an important risk factor of AS that induces disordered lipid metabolism by cutting low-density lipoprotein receptor (4), and participates in the emergence and progression of AS through the non-lipid metabolic pathway, resulting in vascular endothelial cell death and vascular inflammation (5). However, the underlying mechanisms remain unclear.

Programmed cell death, such as autophagy, apoptosis and pyroptosis, occurs in pathological and disease progression. Cell pyroptosis is accompanied by the release of inflammatory mediators, such as interleukin (IL)-1 $\beta$ and IL-18 $(6,7)$, which can induce cell dysfunction and plaque formation (8). Oxidized low-density lipoprotein (oxLDL), a risk factor for the development of AS, can cause mitochondrial dysfunction and increase reactive oxygen species (ROS) levels in human umbilical vein endothelial cells (HUVECs), thereby inducing the pyroptosis of endothelial cells and the release of inflammatory mediators (9). However, the involvement of PCSK9 in the pyroptosis of vascular endothelial cells remains unclear.

As the energy supplying organelle, the mitochondria regulate cell functions and adjust homeostasis by releasing related signal molecules (10). Homeostasis disruption causes mitochondrial dysfunction, which reduces mitochondrial 
membrane potential, decreases ATP level, and increases ROS production, leading to cell dysfunction. oxLDL can damage mitochondrial function, induce ROS release and cause endothelial cell pyroptosis (9). ROS are important mediators in the signaling pathway of pyroptosis. Abnormal shear stress can promote PCSK9 expression in endothelial cells (11), and PCSK9 upregulation increases ROS levels (12). Therefore, PCSK9 possibly causes pyroptosis. The present study aimed to determine whether PCSK9 regulates the pyroptosis of vascular endothelial cells through the ROS pathway.

\section{Materials and methods}

Cell lines and cell culture. HUVECs (Science Cell Research Laboratories) were cultured in endothelial cell medium (Science Cell Research Laboratories) at $37^{\circ} \mathrm{C}$ with $5 \% \mathrm{CO}_{2}$. The small-interfering RNA (siRNA) kit was purchased from Guangzhou RiboBio Co., Ltd. In brief, HUVECs at a density of $30-50 \%$ were transfected with siRNA (RiboBio Transfection reagent, $50 \mu \mathrm{M}$ ) (siPCSK9, 3'-GAGGTGTATCTCCTAGACA-5'; siUQCRC1, 3'-GTG CACACTTTGTCTGTGA-5') or lentivirus (LV)-PCSK9 (Shanghai GeneChem Co., Ltd.; with Genechem HitransG, $\mathrm{MOI}=50$ ) in the presence or absence of PCSK9 and then incubated with oxLDL (100 $\mu \mathrm{g} / \mathrm{ml}$; Yiyuan Biotechnology Co., Ltd.) for $24 \mathrm{~h}$ in an incubator.

Detection of cell pyroptosis. Lactate dehydrogenase (LDH) release was measured with an LDH kit (Jinagcheng bioengineering institute, Nanjing, China) and Hoechst 33342/propidium iodide (PI) staining were used to evaluate pyroptosis. For LDH release detection, $25 \mu \mathrm{l}$ of cell supernatant and $25 \mu \mathrm{l}$ of substrate were incubated at $37^{\circ} \mathrm{C}$ for $15 \mathrm{~min}$. Subsequently, 2,4-dinitrophenylhydrazine $(25 \mu \mathrm{l})$ and $0.4 \mathrm{~mol} / \mathrm{l} \mathrm{NaOH}$ solution (250 $\mu \mathrm{l})$ (Nanjing Jinagcheng Bioengineering Institute) were added. Following incubation at room temperature for $5 \mathrm{~min}$, the absorbance was determined using a spectrophotometric microplate reader (iMark-03; Bio-Rad Laboratories, Inc.).

For Hoechst 33342/PI staining, the HUVECs were first washed thrice with phosphate-buffered saline (PBS), followed by the addition of $10 \mu \mathrm{l}$ of Hoechst 33342 solution, and incubation at $37^{\circ} \mathrm{C}$ in the dark for $10 \mathrm{~min}$. After removing the dye, the cells were washed times with PBS, added with $5 \mu \mathrm{l}$ of PI dye, and then incubated at $37^{\circ} \mathrm{C}$ in the dark for $10 \mathrm{~min}$. After being washed with PBS 3 times, the cells were observed under a fluorescence microscope (IX3; Olympus Corporation).

$R N A$ extraction and reverse transcription-quantitative $P C R$ (RT-qPCR). TRIzol reagent (Roche Diagnostics) was used to extract total RNA, which was then reverse transcribed into cDNA using the RT kit (FSQ-101, Toyobo Co., Ltd.). The product was processed with SYBR-Green Premix Dimer Eraser (Takara Biotechnology Co., Ltd.), and the level of mRNA was then measured using the ABI 7000 sequence detection system (ABI PRISM $^{\circledR} 7000$, Thermo Fisher Scientific, Inc.) (1 cycle, $95^{\circ} \mathrm{C}, 15 \mathrm{~min} ; 40$ cycles, $\left.95^{\circ} \mathrm{C}, 10 \mathrm{sec}, 60^{\circ} \mathrm{C}, 32 \mathrm{sec}\right) . \mathrm{GAPDH}$ was used as the housekeeping gene for normalization. The primers and sequences used were as follows: IL-1 $\beta$ forward, 5'-CGATCACTGAACTGCACGCT-3' and reverse, 5'-AGA ACACCACTTGTTGCTCCA-3'; GAPDH forward, 5'-AAG
ATCAAGATCATTGCTCCTCCTG-3' and reverse, 5'-GCC GGACTCGTCATACTCCT-3'; and PCSK9 forward, 5'-ACG ATGCCTGCCTCTACTCC-3 and reverse, 5'-GCCTGTGAT GTCCCACTCTGT-3'.

Enzyme-linked immunosorbent assay (ELISA). ELISA kit (NeoBioscience, HongKong) was employed to measure the concentration of IL-1 $\beta$ secreted by HUVECs. Cells were first seeded into 24-well plates. Following the different treatments (oxLDL with/without PCSK9 siRNA, LV-PCSK9), the supernatant $(100 \mu \mathrm{l})$ was collected for ELISA in accordance with the manufacturer's protocol (NeoBioscience Technology Company).

Western blot analysis. Cells were first washed thrice with cold PBS and then lysed with radioimmunoprecipitation assay buffer and phenylmethanesulfonyl fluoride (v/v, 94:6) on ice for $30 \mathrm{~min}$. Lysed samples were centrifuged at $9,500 \mathrm{x}$ g for $15 \mathrm{~min}$ at $4^{\circ} \mathrm{C}$. The concentration of total proteins was determined using the BCA kit (Beijing CWBio). Protein samples (20 $\mu \mathrm{g} / \mathrm{well})$ were loaded for $8-12 \%$ sodium dodecyl sulfate polyacrylamide gel electrophoresis and then transferred onto a nitrocellulose filter membrane, which was blocked with 5\% skimmed milk for $2 \mathrm{~h}$ at room temperature. Subsequently, the membrane was incubated with caspase-1 antibody $(1: 500 ; 22915-1-A P$; ProteinTech Group, Inc.), IL-1 $\beta$ antibody (1:500; 16806-1-AP; ProteinTech Group, Inc.), IL-18 antibody (1:1,000; 10663-1-AP; ProteinTech Group, Inc.), NLRP3 antibody (1:500; A5652; ABclonal Biotech Co., Ltd.), GAPDH antibody (1:1,000; 10494-1-AP; ProteinTech Group, Inc.), PCSK9 antibody (1:500; 55206-1-AP; ProteinTech Group, Inc.), gasdermin D (GSDMD-N; 1:500; ab253350, Abcam), cleaved caspase-1 (1:1,000; ab207802; Abcam) and ubiquinol-cytochrome $c$ reductase core protein 1 (UQCRC1) antibody (1:2,000; 21705-1-AP; ProteinTech Group, Inc.) diluted by the mixture of Tris-buffered saline (containing Tween-20) and $2.5 \%$ skimmed milk buffer at $4^{\circ} \mathrm{C}$ overnight. After washing 5 times with PBS containing Tween-20, the membrane was incubated with fluorescence-conjugated antirabbit $\operatorname{IgG}$ secondary antibody (1:2,000, SA00001-2; ProteinTech Group, Inc.) at room temperature for $2 \mathrm{~h}$. After washing 5 times with Tris-buffered saline with Tween-20 (TBST; \#9997; Cell Signaling Technology, Inc.), the optical density of the related bands was examined using Quantity One software V4.6.6 (Bio-Rad Laboratories, Inc.) with GAPDH as the internal reference.

Immunofluorescence. Following stimulation with various concentrations of oxLDL (50 and $100 \mu \mathrm{g} / \mathrm{ml}$ ) for $24 \mathrm{~h}$, the cells were fixed with $4 \%$ formaldehyde for $30 \mathrm{~min}$. Following incubation with $0.1 \%$ Triton $\mathrm{X}-100$ at room temperature for $30 \mathrm{~min}$, the cells were blocked with goat serum for $1 \mathrm{~h}$ at room temperature. After being washed thrice with PBS, the cells were incubated with diluted PCSK9 antibody (1:100; \#85813, Cell Signaling Technology, Inc.) at $4^{\circ} \mathrm{C}$ overnight. After being washed 5 times with PBS, the cells were incubated with Alexa Fluor-conjugated secondary antibody (1:50, A32731, Invitrogen; Thermo Fisher Scientific, Inc.) in the dark for $1 \mathrm{~h}$ at room temperature. Finally, the nuclei were stained with 4',6-diamidino-2-phenylindole (Beyotime Institute of Biotechnology, Inc.) for $5 \mathrm{~min}$ at room temperature. After being washed 5 times with PBS, the cells were observed under a fluorescence microscope (IX3; Olympus Corporation). 
Determination of ATP concentration incells.Cells (1.0x10\% were washed with PBS twice and then lysed with $150 \mathrm{ml}$ of lysis buffer. After being centrifuged at $1,500 \mathrm{x}$ g for $5 \mathrm{~min}$ at $4^{\circ} \mathrm{C}, \mathrm{ATP}$ detection reagent (Nanjing Jinagcheng Bioengineering Institute) was added, followed by examination using a microplate reader (iMark-03; Bio-Rad Laboratories, Inc.). ATP concentration was calculated using the standard curve.

Detection of ROS. The mitochondrial ROS probe (DCFH-DA; Nanjing KeyGen Biotech Co. Ltd.) was used to detect ROS production by HUVECs. Upon reaching 30-50\% confluence, the cells were transfected with siRNA against PCSK9 and siUQCRC1. The transfected cells were incubated with oxLDL $(100 \mu \mathrm{g} / \mathrm{ml})$ for $24 \mathrm{~h}$ at room temperature. After discarding the medium, the cells were washed with cold PBS (pH 7.4) and then incubated with DCFH-DA at room temperature for $30 \mathrm{~min}$. After being washed thrice with PBS, the cells were observed under a fluorescence microscope (IX3; Olympus Corporation).

Detection of mitochondrial membrane potential. The cells were first washed with PBS and then centrifuged at 1,500 $\mathrm{x} g$ for $5 \mathrm{~min}$ at room temperature. After discarding the supernatant, the cells were resuspended with $500 \mu \mathrm{l}$ of JC- 1 and then incubated at $37^{\circ} \mathrm{C}$ and $5 \% \mathrm{CO}_{2}$ for $15 \mathrm{~min}$. Following centrifugation at $1,500 \mathrm{x}$ g for $5 \mathrm{~min}$ at room temperature, the supernatant was removed and the cells were resuspended with $500 \mu \mathrm{l}$ of $1 \mathrm{X}$ incubation buffer. The cells were then observed under a fluorescence microscope (IX3; Olympus Corporation) and examined by flow cytometry $(\mathrm{Ex}=488 \mathrm{~nm} ; \mathrm{Em}=530 \mathrm{~nm})\left(\right.$ Sysmex-Partec CyFlow $^{\circledR}$ Cube 6, Sysmex Partec). The ratio of green fluorescence intensity/red fluorescence intensity directly reflected mitochondrial membrane potential and mitochondrial function.

Statistical analysis. All data are presented as the means value with standard deviation. Differences between groups were determined by one-way ANOVA accompanied by the Tukey's or Bonferroni post hoc test (GraphPad Prism version 5.0; GraphPad, Inc.). A value of $\mathrm{P}<0.05$ was considered to indicate a statistically significant difference.

\section{Results}

oxLDL induces HUVEC injury, pyroptosis and the release of inflammatory factors. HUVECs were incubated with oxLDL (50 and $100 \mu \mathrm{g} / \mathrm{ml}$ ) for $24 \mathrm{~h}$ prior to detecting the expression levels of related molecules to explore whether oxLDL can cause damage to and the pyroptosis of these cells. oxLDL upregulated the protein expression levels of NLRP3, caspase-1 and IL-18 (Fig. 1A and B), and also increased the protein and mRNA expression of IL-1 $\beta$ (Fig. 1A-C). GSDMD-N expression was also increased by oxLDL stimulation (Fig. S1). The results of ELISA revealed that the IL- $1 \beta$ protein content increased in the supernatant of the HUVECs stimulated with oxLDL (Fig. 1D). The LDH level was also measured to determine the damage caused to the HUVECs by oxLDL. The data indicated that oxLDL promoted the release of LDH in a dose-dependent manner (Fig. 1E). Double staining with Hoechst 33342 and PI revealed that the ratio of PI-positive cells increased with the increasing oxLDL concentration (Fig. $1 F$ and G). These results suggest that oxLDL induces injury to, and the pyroptosis of
HUVECs, as well as the release of inflammatory factors from HUVECs.

oxLDL upregulates PCSK9 protein expression in HUVECs. PCSK9 expression was examined following stimulation with oxLDL to examine the association between oxLDL and PCSK9. Western blot analysis revealed that oxLDL stimulation upregulated PCSK9 expression in HUVECs (Fig. 2A and B). Immunofluorescence also revealed similar findings, in which the PCSK9 fluorescence intensity increased following stimulation with oxLDL (Fig. 2C and D). These results indicate that oxLDL upregulates PCSK9 protein expression in HUVECs.

siPCSK9 suppresses the damage, release of inflammatory factors and pyroptosis of HUVECs induced by oxLDL. It has been demonstrated that PCSK9 inhibition prevents inflammation and alleviates cell death (13). In the present study, the level of pyroptosis in the HUVECs was detected following stimulation with oxLDL with or without siPCSK9 transfection (50 $\mu \mathrm{M}$ siRNA). The siRNA efficiency of PCSK9 was determined (Fig. 3A). The expression levels of NLRP3, caspase-1 and IL-18 in the oxLDL group were significantly higher than those in the control group and siPCSK9 group (Fig. 3B and C). In addition, transfection with siPCSK9 decreased the protein and mRNA expression levels of IL-1 $\beta$ in the oxLDL group (Fig. 3B-D). The results of ELISA and $\mathrm{LDH}$ assay revealed that PCSK9 interference attenuated the upregulating effect of oxLDL on IL-1 $\beta$ expression (Fig. 3E) and reduced the damage to HUVECs caused by oxLDL, evidenced by the decreased activity of LDH (Fig. 3F). The PI staining data revealed that the pyroptosis of HUVECs was reduced by PCSK9 interference (Fig. $3 \mathrm{G}$ and $\mathrm{H}$ ). These results suggest that PCSK9 interference suppresses the oxLDL-induced damage, the release of inflammatory substances and the pyroptosis of HUVECs.

Overexpression of PCSK9 induces the pyroptosis of HUVECs. HUVECs were transfected with LV-PCSK9 to explore the association between PCSK9 and pyroptosis. The mRNA and protein levels of PCSK9 significantly increased following transfection with LV-PCSK9 (Fig. 4A and B). In addition, NLRP3, caspase-1 cleaved caspase- 1 and IL-1 $\beta$ protein expression increased following the overexpression of PCSK9 (Fig. 4C and D). The protein and mRNA expression of IL-1 $\beta$ (Fig. 4E and F) and the LDH level (Fig. 4G) in the supernatant increased following the overexpression of PCSK9. In addition, the ratio of PI-positive cells significantly increased following the overexpression of PCSK9 (Fig. 4H and I). These results indicate that PCSK9 overexpression induces the pyroptosis of HUVECs.

PCSK9 overexpression increases ROS generation and induces mitochondrial dysfunction by inhibiting UQCRC1. Excessive ROS production caused by mitochondrial dysfunction can promote the pyroptosis of endothelial cells (9) and reduce ATP production (14). In the present study, the intracellular ROS levels were detected following transfection with LV-PCSK9 to determine whether PCSK9 promotes the pyroptosis of endothelial cells through the ROS pathway. PCSK9 overexpression elevated the ROS levels (Fig. 5A and B) and 
A
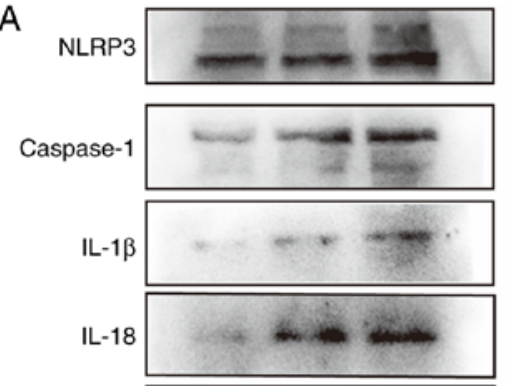

GAPDH

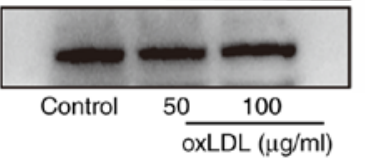

B

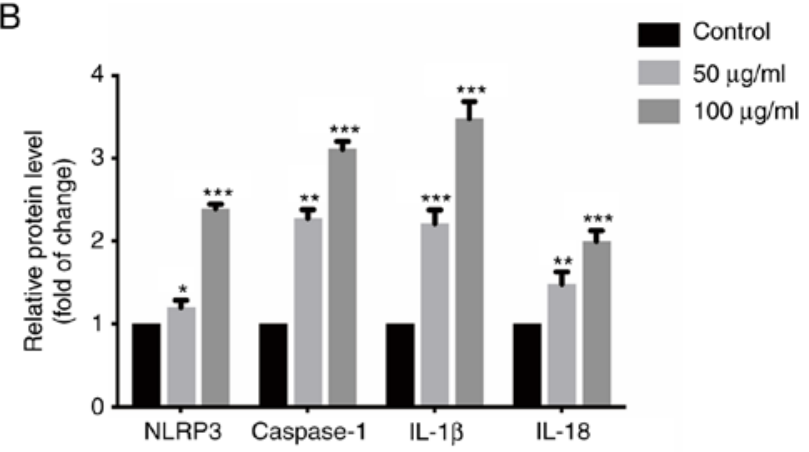

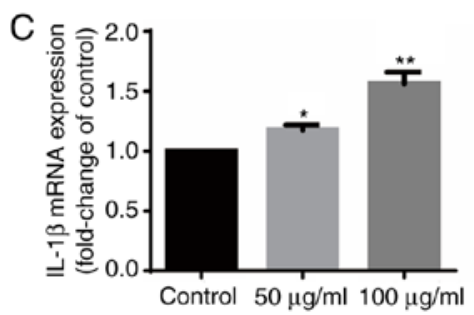
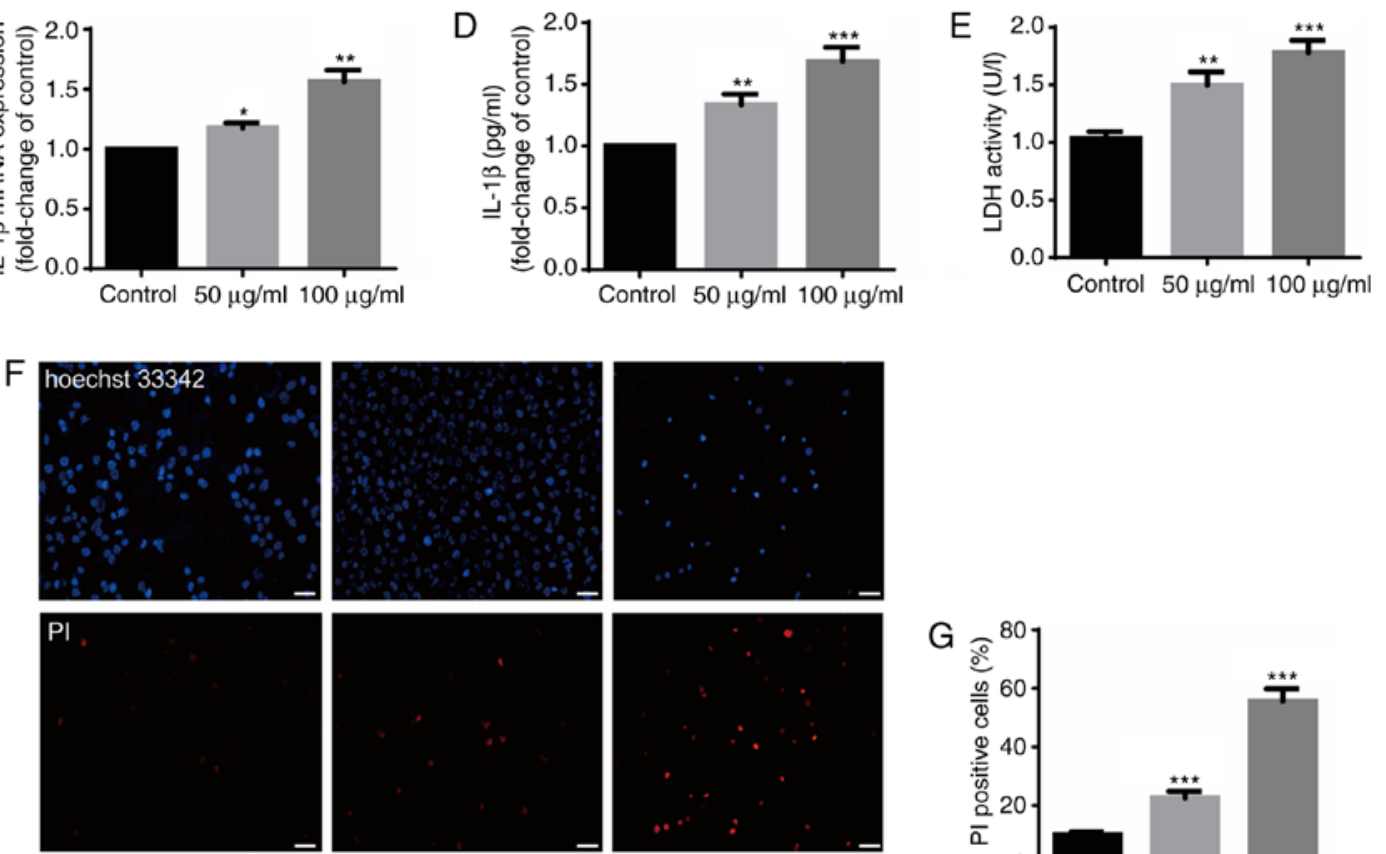

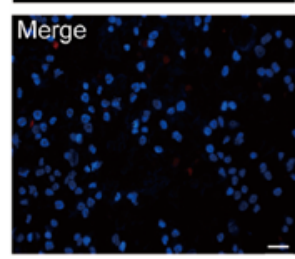

Control

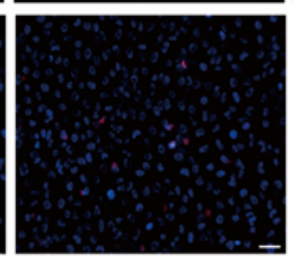

50

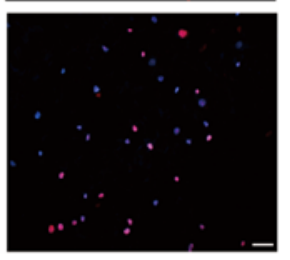

100

$\operatorname{oxLDL}(\mu \mathrm{g} / \mathrm{ml})$

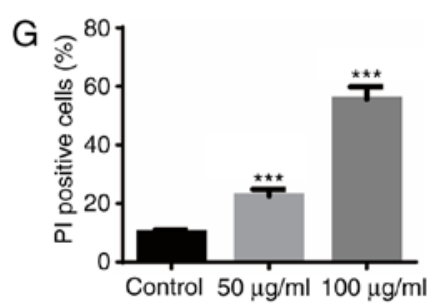

Figure 1. oxLDL induces the pyroptosis of HUVECs. (A and B) Expression of NLRP3, caspase-1, IL-1 $\beta$ and IL-18 proteins following stimulation with 50 or $100 \mu \mathrm{g} / \mathrm{ml}$ oxLDL for $24 \mathrm{~h}$. Western blot analysis was used to measure protein expression. ${ }^{*} \mathrm{P}<0.05,{ }^{* *} \mathrm{P}<0.01$ and ${ }^{* * * *} \mathrm{P}<0.001$ compared with the control group. (C) Expression of IL-1 $\beta$ mRNA in HUVECs stimulated with 50 or $100 \mu \mathrm{g} / \mathrm{ml}$ oxLDL for $24 \mathrm{~h}$. "P<0.05 and ** P<0.01 compared with the control group. (D) Concentration of IL-1 $\beta$ protein in supernatant of HUVECs stimulated with 50 or $100 \mu \mathrm{g} / \mathrm{ml}$ oxLDL for $24 \mathrm{~h}$. ELISA was used to measure protein concentration. ${ }^{* *} \mathrm{P}<0.01$ and ${ }^{* * *} \mathrm{P}<0.001$ compared with the control group. (E) LDH levels in the supernatant of HUVECs stimulated with 50 or $100 \mu \mathrm{g} / \mathrm{ml}$ oxLDL for 24 h. ${ }^{* *} \mathrm{P}<0.01$ and ${ }^{* * *} \mathrm{P}<0.001$ compared with the control group. (F and G) Ratio of PI-positive HUVECs stimulated with 50 or $100 \mu \mathrm{g} / \mathrm{ml}$ oxLDL for $24 \mathrm{~h}$. Propidium iodide (PI, red color). Original magnification, $\mathrm{x} 100$; scale bar, $100 \mu \mathrm{m} .{ }^{* * *} \mathrm{P}<0.001$ compared with the control group. All results are expressed as the means \pm SD of 3 independent experiments. oxLDL, oxidized low-density lipoprotein; HUVECs, human umbilical cord endothelial cells; NLRP3, NLR family pyrin domain containing 3 .

inhibited ATP production (Fig. 5C). UQCRC1 is a subunit of mitochondrial respiratory chain complex III and maintains mitochondrial function (14). UQCRC1 expression was thus examined following the overexpression of PCSK9 in order to determine the association between PCSK9 and UQCRC1. The data indicated that PCSK9 overexpression inhibited the protein expression of UQCRC1 (Fig. 5D). These results suggest that
PCSK9 increases ROS production and causes mitochondrial disorder by inhibiting UQCRC1.

siPCSK 9 reverses mitochondrial dysfunction induced by oxLDL by recovering UQCRCl expression. HUVECs were incubated with siPCSK9 for $24 \mathrm{~h}$ and then stimulated with oxLDL $(100 \mu \mathrm{g} / \mathrm{ml})$ to investigate whether oxLDL inhibits 
A

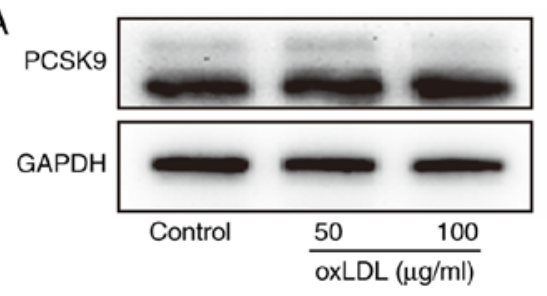

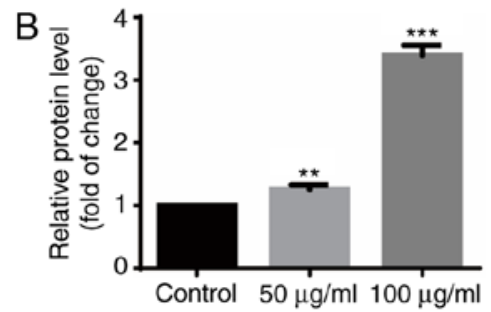
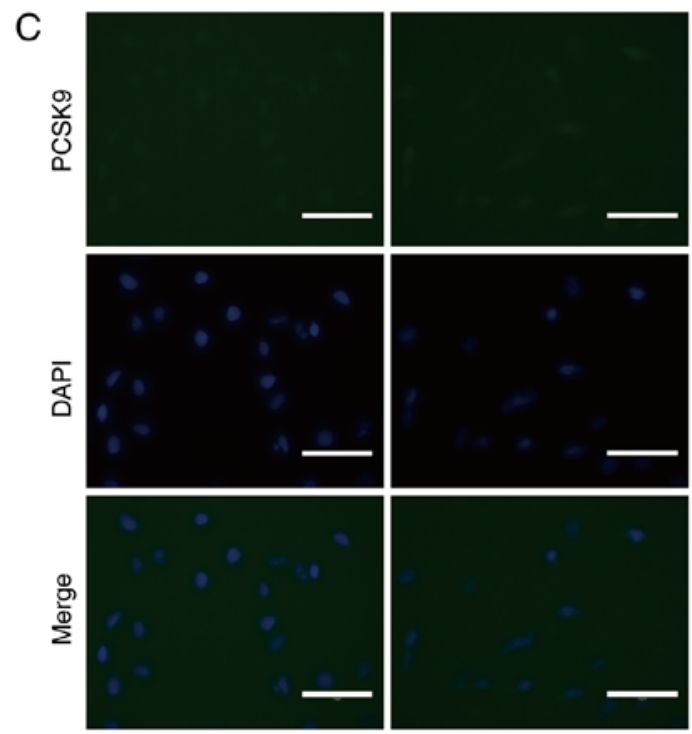

Control

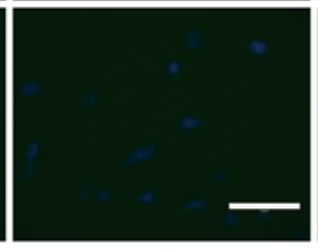

50
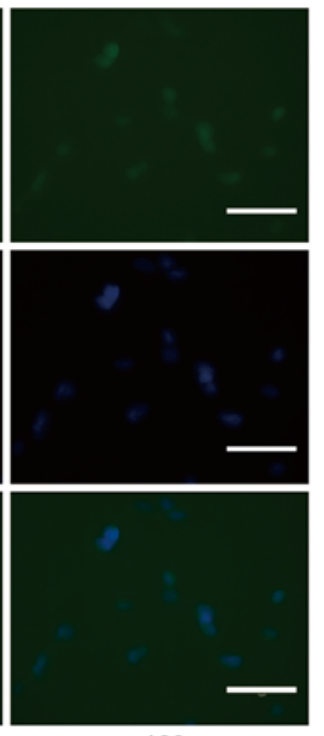

100

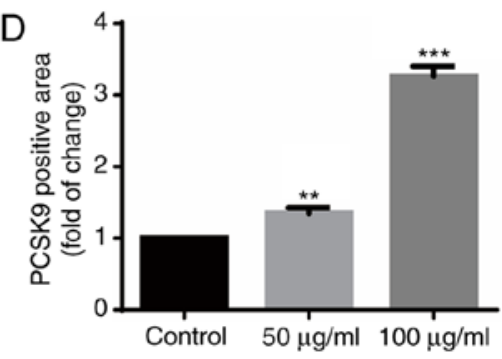

$\operatorname{oxLDL}(\mu \mathrm{g} / \mathrm{ml})$

Figure 2. oxLDL upregulates the expression of PCSK9 protein in HUVECs. (A and B) Expression of PCSK9 protein following stimulation with 50 or $100 \mu \mathrm{g} / \mathrm{ml}$ oxLDL for $24 \mathrm{~h}$. Western blot analysis was used to measure protein expression. ${ }^{* *} \mathrm{P}<0.01$ and ${ }^{* * *} \mathrm{P}<0.001$ compared with the control group. (C and D) Fluorescence intensity of PCSK9 in HUVECs stimulated with 50 or $100 \mu \mathrm{g} / \mathrm{ml}$ oxLDL. The nuclei were stained blue with 4',6-diamidino-2-phenylindole (DAPI). Original magnification, $\mathrm{x} 100$; scale bar, $75 \mu \mathrm{m}$. ${ }^{* *} \mathrm{P}<0.01$ and ${ }^{* * * *} \mathrm{P}<0.001$ compared with control group. All results are expressed as the means \pm SD of 3 independent experiments. oxLDL, oxidized low-density lipoprotein; HUVECs, human umbilical cord endothelial cells; PCSK9, proprotein convertase subtilisin/kexin type 9 .

UQCRC1 expression through PCSK9. The data indicated that UQCRC1 expression was significantly increased following transfection with siPCSK9 compared with that after oxLDL stimulation alone (Fig. 6A). The ROS level significantly decreased following transfection with siPCSK9, but was significantly increased following transfection with siPCSK9 and siUQCRC1 (the transfection efficiency is shown in Fig. 6B and C) compared with that following transfection with siPCSK9 only (Fig. 6D and E). The cell membrane potential level was also examined by JC-1 staining. The green fluorescence was higher in the oxLDL group than in the control group and recovered compared with that in the siPCSK9-transfected group. However, transfection with siPCSK9 and siUQCRC1 enhanced the intensity of green fluorescence (Fig. 6F). Flow cytometry revealed that the area in the Q2 quadrant decreased, whereas the areas in the Q3 and Q4 quadrants increased following oxLDL stimulation. PCSK9 interference increased the area in Q2 quadrant. The simultaneous interference of PCSK9 and UQCRC1 decreased the area in the Q2 quadrant, but increased the areas in the Q3 and Q4 quadrants (Fig. 6F and G). These results indicate that oxLDL inhibits UQCRC1 expression, promotes mitochondrial membrane potential collapse, and damages mitochondrial function, and these processes can be reversed PCSK9 interference.

\section{Discussion}

The present study demonstrated that PCSK9 interference attenuated HUVEC pyroptosis induced by oxLDL. On the one hand, oxLDL induced the pyroptosis of HUVECs in a concentration-dependent manner and upregulated PCSK9 expression. PCSK9 interference relieved the pyroptosis induced by oxLDL. On the other hand, PCSK9 overexpression promoted pyroptosis, UQCRC1 downregulation and ROS generation. siPCSK9 can alleviate the inhibition of UQCRC1 expression by oxLDL, ROS production, and the disorder of mitochondrial membrane potential (Fig. 7).

$\mathrm{AS}$ is a chronic inflammatory disease accompanied by endothelial dysfunction and lipid deposition (1). Endothelial dysfunction is the initial cause of AS. In AS lesions, endothelial cells are often accompanied by pyroptosis (15), which is associated with the activation of related inflammatory bodies (NLRP3 and caspase-1) and the release of inflammatory factors (IL-1 $\beta$ and IL-18) and cell dysfunction to stimulate the recruitment of immune cells and then promote AS (16). However, the mechanisms underlying pyroptosis remain unclear. NLRP3 and pyrin in cells can recruit inflammatory bodies to form mature bodies, thus leading to the maturation of inflammatory factors and the activation of gasdermin family (17). ROS can activate NLRP3 and mediate pyroptosis (18). In the 
A

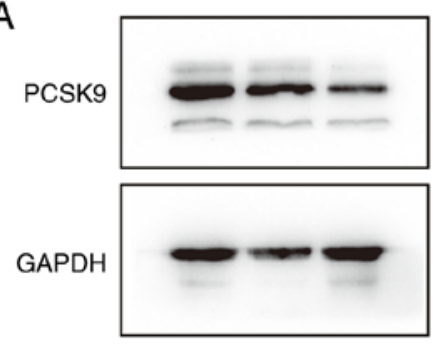

Control siNC siPCSK9

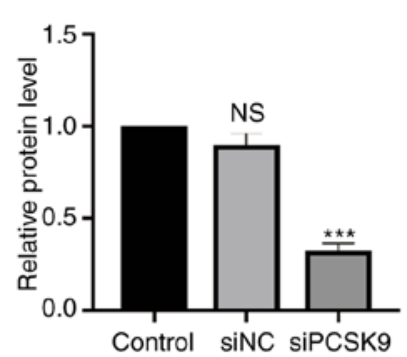

Control siNC siPCSK9
B

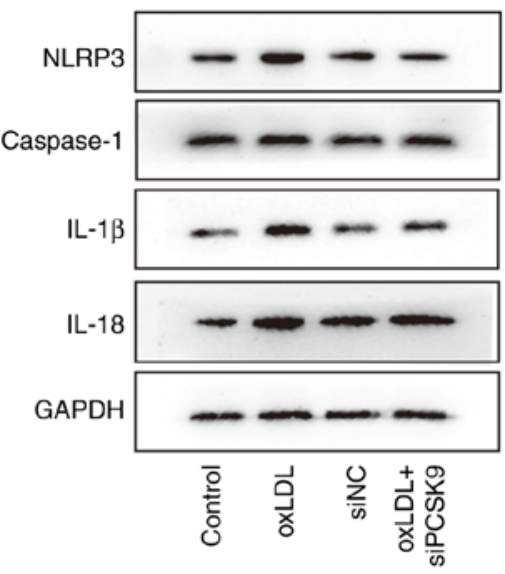

E

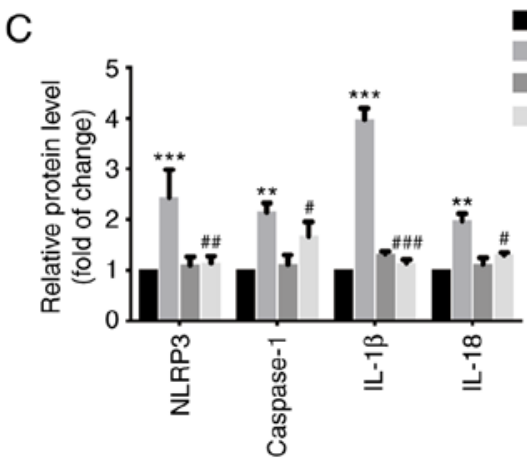

F

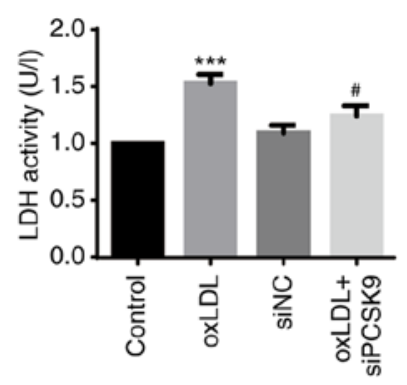

Control

oxLDL

SiNC

siPCSK9

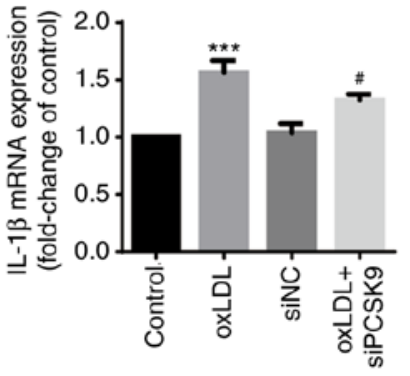

$G$ hoochsit 33342 .
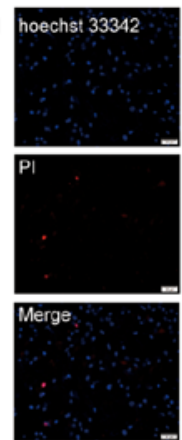

Control
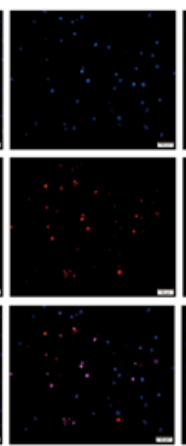

oxLDL
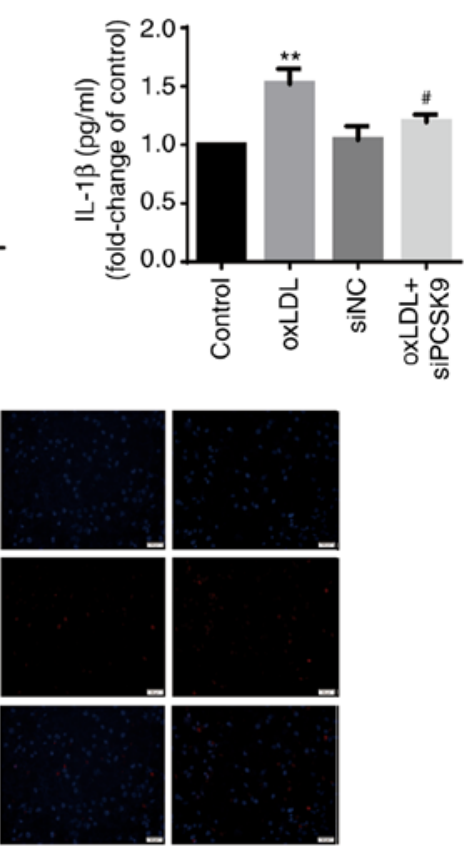

siNC

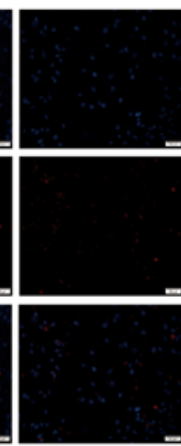

oxLDL+

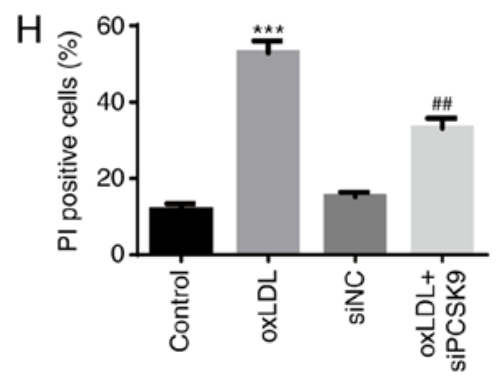

Figure 3. Interference with PCSK9 inhibits the oxLDL-induced pyroptosis of HUVECs. (A) Expression of PCSK9 protein in HUVECs following interference with PCSK9 $(50 \mathrm{nM}, 24 \mathrm{~h})$ Western blot analysis was used to measure protein expression. ${ }^{* * * *} \mathrm{P}<0.001$ compared with the control group; NS, not significant. (B and C) Expression of NLRP3, caspase-1, IL-1 $\beta$ and IL-18 protein in HUVECs following interference with PCSK9 following stimulation with $100 \mu \mathrm{g} / \mathrm{ml}$ oxLDL for $24 \mathrm{~h}$. Western blot analysis was used to measure protein expression. ${ }^{* *} \mathrm{P}<0.01$ and ${ }^{* * * *} \mathrm{P}<0.001$ compared with the control group; ${ }^{\# \mathrm{P}}<0.05$, ${ }^{\# \#} \mathrm{P}<0.01$ and ${ }^{\# \# \#} \mathrm{P}<0.001$ compared with the oxLDL group. (D) Expression of IL-1 $\beta$ mRNA. ${ }^{* * *} \mathrm{P}<0.001$ compared with the control group; ${ }^{*} \mathrm{P}<0.05$ compared with the oxLDL group. (E) Concentration of IL-1 $\beta$ in supernatant of HUVECs. ${ }^{* *} \mathrm{P}<0.01$ compared with the control group; ${ }^{*} \mathrm{P}<0.05$ compared with the oxLDL group. $(\mathrm{F}) \mathrm{LDH}$ levels in supernatant of HUVECs. ${ }^{* * * *} \mathrm{P}<0.001$ compared with the control group; ${ }^{*} \mathrm{P}<0.05$ compared with the oxLDL group. (G and $\left.\mathrm{H}\right)$ Ratio of PI-positive HUVECs. Original magnification, $\mathrm{x} 100$; scale bar, $100 \mu \mathrm{m} .{ }^{* * *} \mathrm{P}<0.001$ compared with the control group; ${ }^{\# \#} \mathrm{P}<0.01$ compared with the oxLDL group. All results are expressed as the means \pm SD of 3 independent experiments. oxLDL, oxidized low-density lipoprotein; HUVECs, human umbilical cord endothelial cells; PCSK9, proprotein convertase subtilisin/kexin type 9.

present study, PCSK9 overexpression induced pyroptosis and increased ROS production.
PCSK9 is involved in the pathogenesis of AS by regulating lipid metabolism $(19,20)$ as confirmed by the clinical 

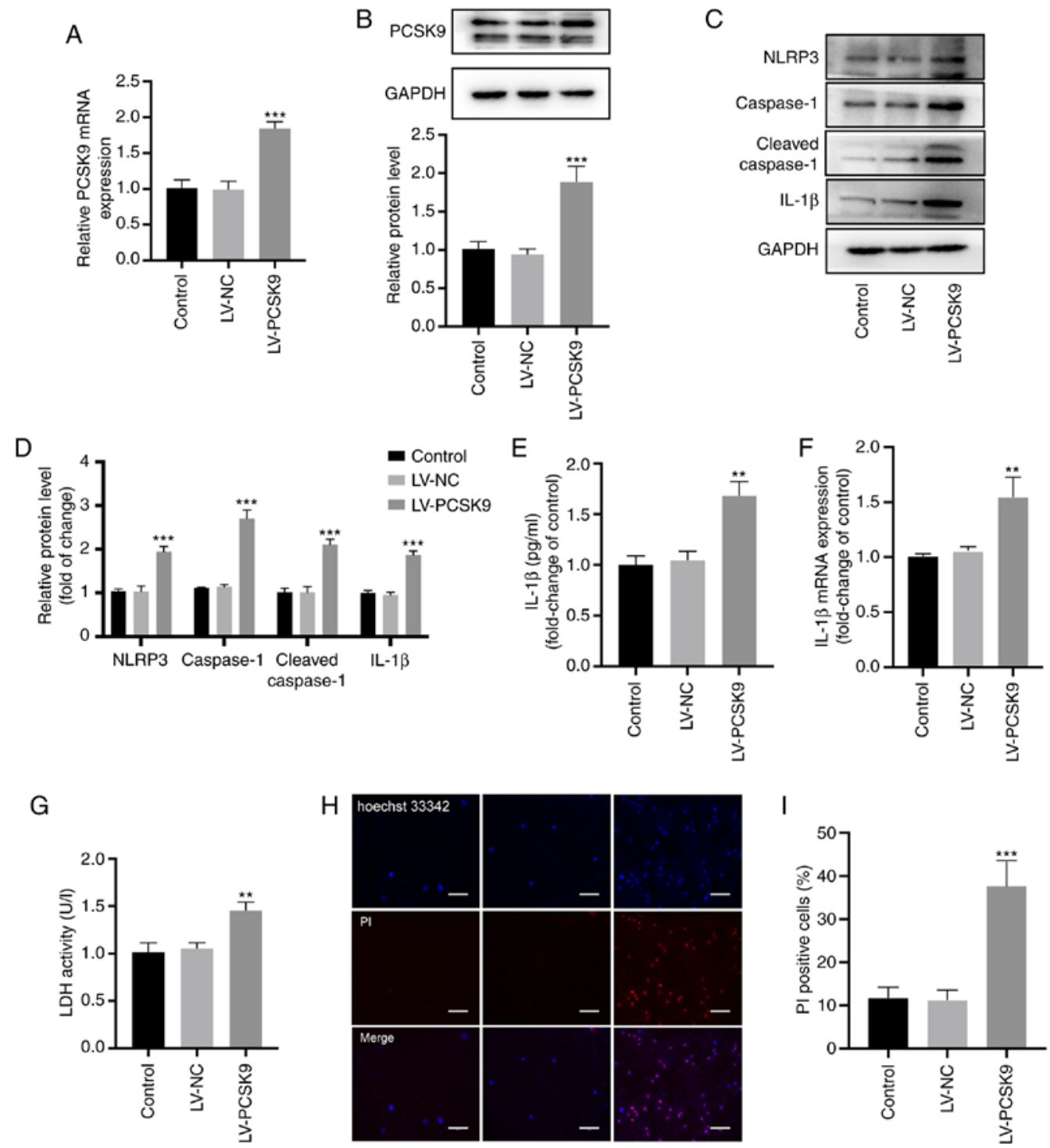

Figure 4. Overexpression of PCSK9 induces the pyroptosis of HUVECs. (A and B) Expression of PCSK9 (A) mRNA and (B) protein in HUVECs following transfection with LV-PCSK9. ${ }^{* * *} \mathrm{P}<0.001$ compared with LV-NC group. (C and D) Expression of NLRP3, caspase-1 and IL-1 $1 \beta$ proteins in HUVECs. ${ }^{* * *} \mathrm{P}<0.001$ compared with the LV-NC group. (E) Concentration of IL- $1 \beta$ in the supernatant of HUVECs determined by ELISA. ${ }^{* *} \mathrm{P}<0.01$ compared with the LV-NC group. (F) Expression of IL-1 $\beta$ mRNA in HUVECs. ${ }^{* *} \mathrm{P}<0.01$ compared with the LV-NC group. (G) LDH levels in the supernatant of HUVECs. ${ }^{* *} \mathrm{P}<0.01$ compared with the LV-NC group. (H and I) Ratio of PI-positive HUVECs. PI is shown in red. Original magnification, x100; scale bar, $100 \mu \mathrm{m} .{ }^{* * * *} \mathrm{P}<0.001 \mathrm{compared}$ with the LV-NC group. All results are expressed as the means \pm SD of 3 independent experiments. oxLDL, oxidized low-density lipoprotein; HUVECs, human umbilical cord endothelial cells; PCSK9, proprotein convertase subtilisin/kexin type 9.
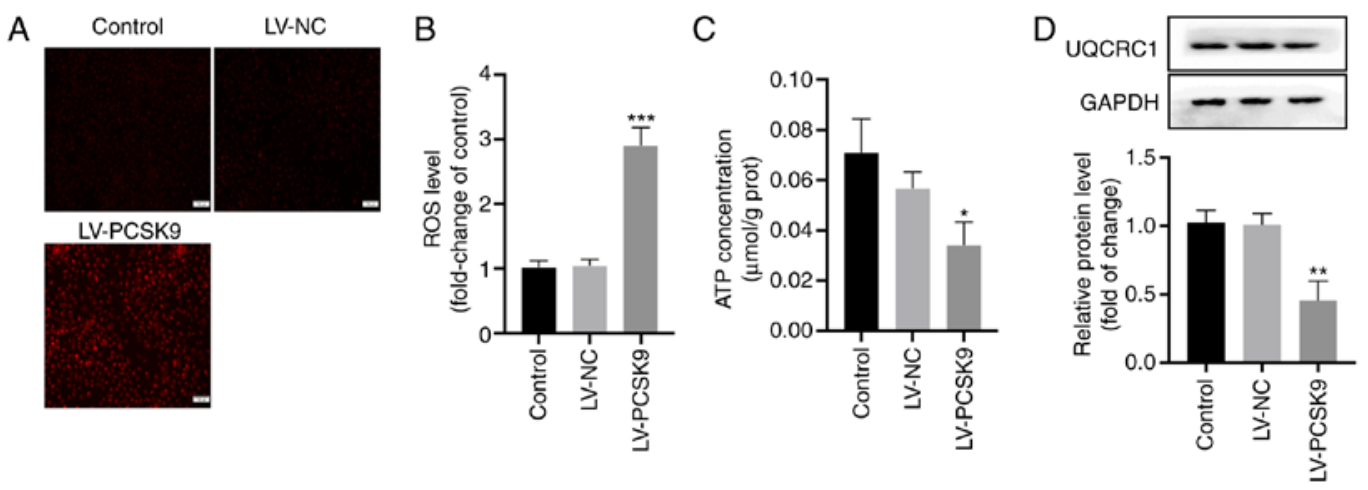

Figure 5. Overexpression of PCSK9 increases ROS production and inhibits ATP production. (A and B) ROS level in HUVECs following transfection with LV-PCSK9. Original magnification, $\mathrm{x} 100$; scale bar, $100 \mu \mathrm{m}$. ${ }^{* * * *} \mathrm{P}<0.001$ compared with the LV-NC group. (C) ATP concentration in HUVECs following transfection with LV-PCSK9. "P<0.05 compared with the LV-NC group. (D) Expression of UQCRC1 protein in HUVECs following transfection with LV-PCSK9. * P $<0.01$ compared with the LV-NC group. All results are expressed as the means \pm SD of 3 independent experiments. oxLDL, oxidized low-density lipoprotein; HUVECs, human umbilical cord endothelial cells; PCSK9, proprotein convertase subtilisin/kexin type 9; ROS, reactive oxygen species; ATP, adenosine triphosphate. 
A

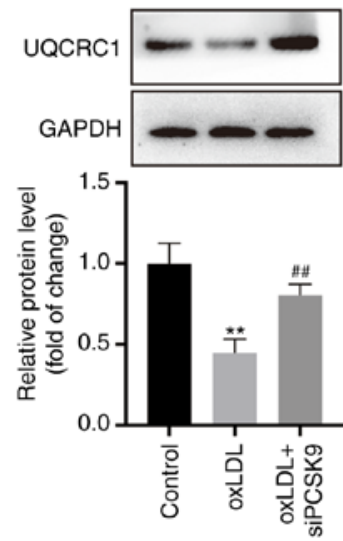

D

$\mathrm{F}$

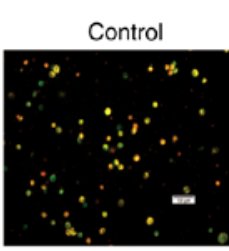

B

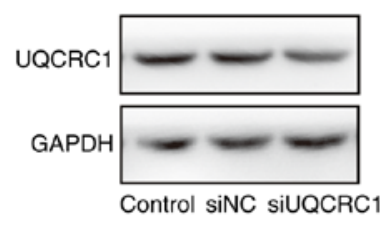

C

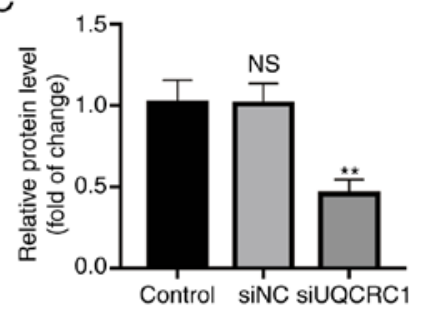

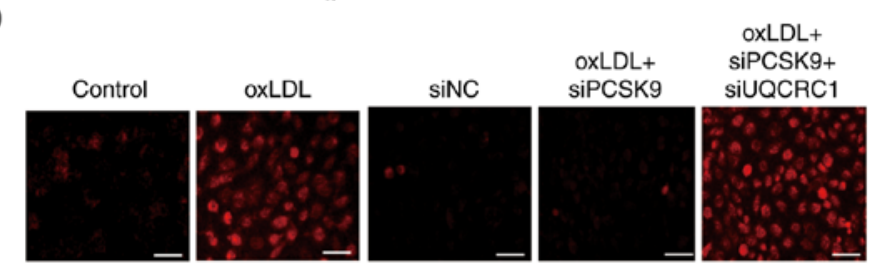
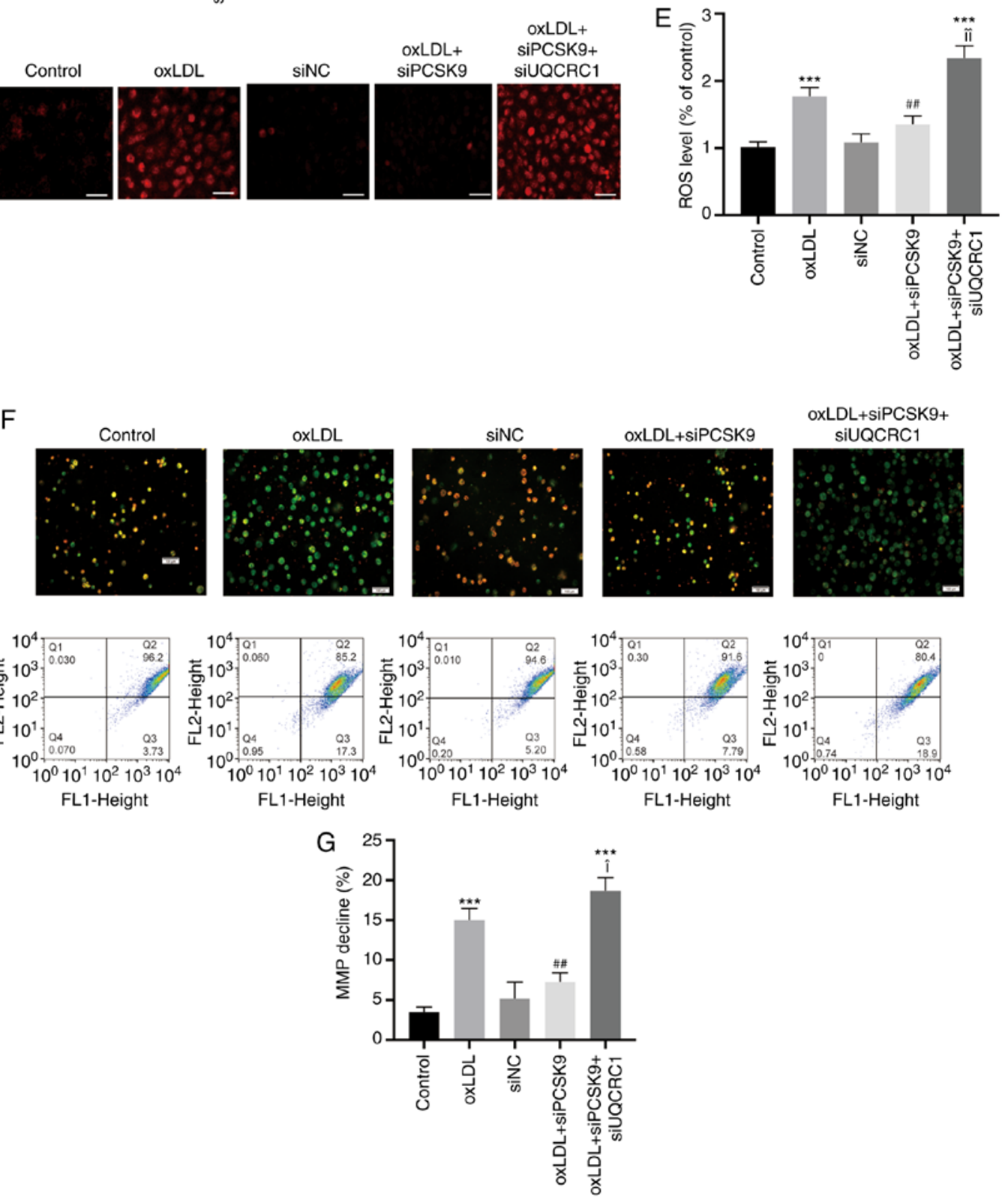

Figure 6. Interference with PCSK9 attenuates excessive production of ROS. (A) Expression of UQCRC1 following stimulation with oxLDL (100 $\mu \mathrm{g} / \mathrm{ml}$ ), and the effect of interference with PCSK9 expression. ${ }^{* *} \mathrm{P}<0.01$ compared with the control group; ${ }^{\# \#} \mathrm{P}<0.01$ compared with the oxLDL group. (B and $\mathrm{C}$ ) Expression of UQCRC1 protein in HUVECs following interference with UQCRC1 $(50 \mathrm{nM}, 24 \mathrm{~h})$. Western blot analysis was used to measure protein expression. ${ }^{* *} \mathrm{P}<0.01$ compared with the control group. (D and E) Mitochondrial ROS level following stimulation with oxLDL (100 $\mu \mathrm{g} / \mathrm{ml})$, and the effect of interference with PCSK9 expression and UQCRC1 expression. Original magnification, $\mathrm{x} 200$; scale bar, $20 \mu \mathrm{m}$. ${ }^{* * *} \mathrm{P}<0.001$ compared with the control group; ${ }^{\# \#} \mathrm{P}<0.01 \mathrm{compared}$ with the oxLDL group; ${ }^{\text {¥¥ }} \mathrm{P}<0.01$ compared with the oxLDL + siPCSK9 group. (F) Changes in mitochondrial membrane potential as observed by fluorescence microscopy. Original magnification, $\mathrm{x} 100$; scale bar, $100 \mu \mathrm{m}$. (G) Mitochondrial membrane potential levels measured by flow cytometry. ${ }^{* * *} \mathrm{P}<0.001 \mathrm{compared}$ with the control group; ${ }^{\# \prime} \mathrm{P}<0.01$ compared with the oxLDL group; ${ }^{\circ} \mathrm{P}<0.05$ compared with the oxLDL + siPCSK9 group. All results are expressed as the means \pm SD of 3 independent experiments. oxLDL, oxidized low-density lipoprotein; HUVECs, human umbilical cord endothelial cells; PCSK9, proprotein convertase subtilisin/kexin type 9; UQCRC1, ubiquinol-cytochrome $c$ reductase core protein 1. 


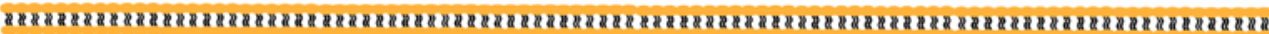

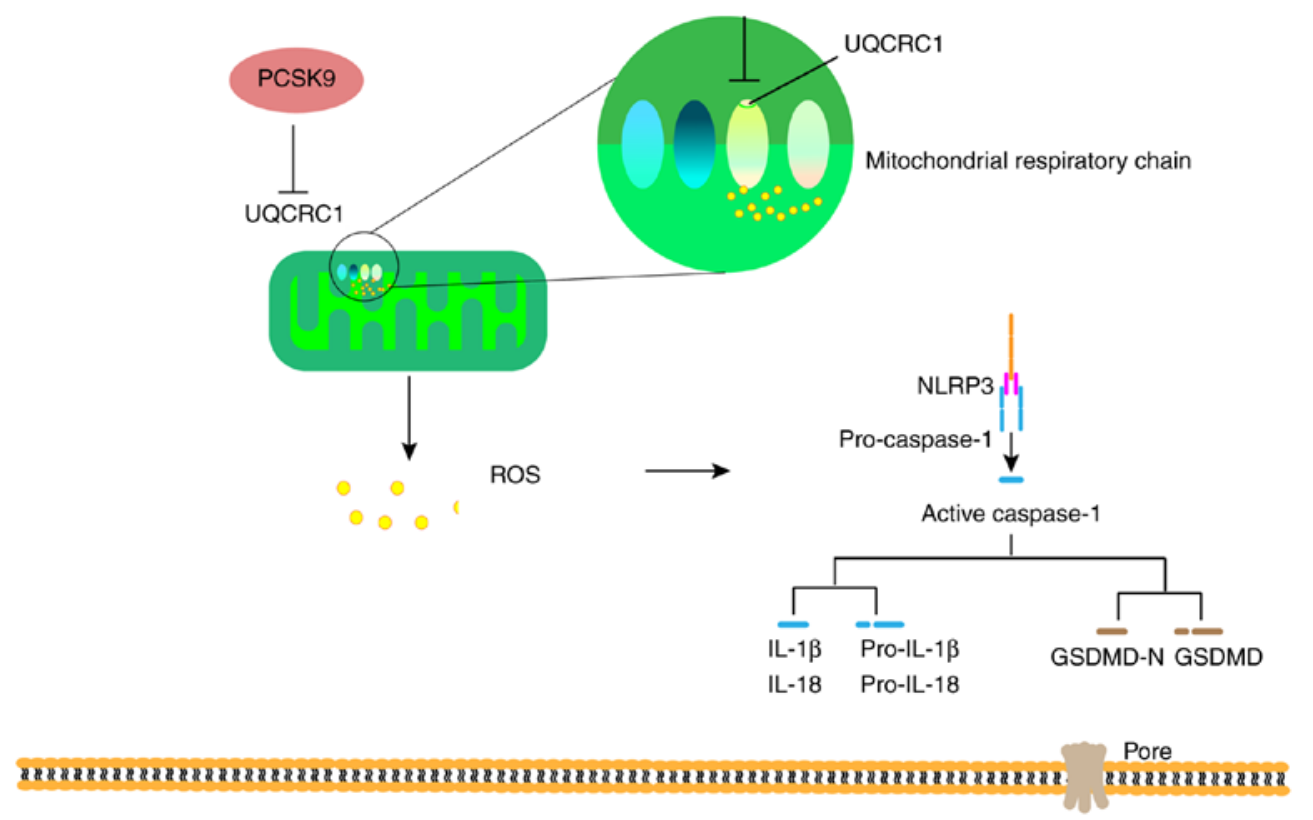

Figure 7. Schematic diagram of the regulatory effects of PCSK9 on endothelial cell pyroptosis by increasing ROS production via the inhibition of UQCRC1. PCSK9 leads to mitochondrial dysfunction through the inhibition of the subunit UQCRC1, and this promotes the production of ROS. ROS improves the action of NLRP3, which recruits caspase-1 and promotes inflammatory cytokine maturation and cellular pore formation. PCSK9, proprotein convertase subtilisin/kexin type 9; UQCRC1, ubiquinol-cytochrome $c$ reductase core protein 1; ROS, reactive oxygen species; NLRP3, NLR family pyrin domain containing 3 .

lipid-lowering effect of PCSK9 inhibitors (21). This enzyme binds to LDLR, promotes its intracellular degradation, and then reduces LDLR level and LDL-C metabolism (19). PCSK9 is also involved in cell death and inflammation. Low shear stress can promote the expression of lectin-like oxidized low-density lipoprotein receptor-1 (LOX-1) by activating ROS and NF- $\mathrm{B}$, and the upregulation of LOX-1 can upregulate PCSK9 expression and vice versa (11). In the present study, PCSK9 overexpression promoted the pyroptosis of endothelial cells, and its interference alleviated the pyroptosis induced by oxLDL. These results suggest that PCSK9 is an important mediating factor in pyroptosis and ROS production. However, the mechanisms through which PCSK9 upregulates ROS expression have been rarely reported, at least to the best of our knowledge.

The mitochondria can regulate cell function by releasing signal molecules (10). Excessive inflammatory stimulation and oxidative stress can promote the disorder of mitochondrial regulation (22). oxLDL inhibits mitochondrial gene expression (14), induces mitochondrial dysfunction, and promotes AS (23). Moreover, PCSK9 is closely related to mitochondrial function and oxidative damage $(24,25)$, promotes the mitochondrial DNA damage of endothelial cells, and facilitates the occurrence of apoptosis (26). However, the association between PCSK9 and mitochondrial damage remains unclear. UQCRC1 is a subunit of mitochondrial complex III, and its interference in endothelial cells can lead to excess ROS production (14). The present study revealed that PCSK9 inhibited the function of mitochondrial complex III by downregulating UQCRC1, leading to ROS elevation. This finding also suggests that PCSK9 is an important mediator of the oxidative damage mediated by oxLDL through mitochondrial-related DNA. Elevated ROS levels have a pathological potential in leading mtDNA mutation and oxidative damage to the respiratory chain (27), which also increased following the overexpression of PCSK9 in the present study. Therefore, the inhibition of UQCRC1 may result from the ROS level regulated by PCSK9. However, ROS elevation also upregulates PCSK9 expression and damages mitochondrial genes (11). Therefore, ROS and PCSK9 can be regulated by mitochondrial genes (such as UQCRC1) and then participate in the occurrence and development of AS.

The present study has some limitations. First, the extracellular level of PCSK9 was not detected under the effect of oxLDL. Second, the mechanism through which PCSK9 inhibits the expression of UQCRC1 was not elucidated. Third, the effect of UQCRC1 defect was only observed in vitro, and in vivo experiments were not conducted. These limitations also provide directions for future studies.

In conclusion, the present study demonstrates that oxLDL promotes the pyroptosis of HUVECs and simultaneously upregulates PSCK9 expression, leading to mitochondrial dysfunction and ROS production resulting from UQCRC1 inhibition. However, PCSK9 interference blocks oxLDL-induced UQCRC1 inhibition, pyroptosis and mitochondrial ROS production in HUVECs. These results indicate that UQCRC1 plays a potential role in the antioxidant response and AS treatment.

\section{Acknowledgements}

Not applicable.

\section{Funding}

The present study was supported by the Natural Science Foundation of China (grant no. 81970389) and the Natural Science Foundation of Hunan Province China (grant nos. 2019JJ50531 and 2020JJ4541). 


\section{Availability of data and materials}

The datasets used and/or analyzed during the current study are available from the corresponding author on reasonable request.

\section{Authors' contributions}

LL and ZW contributed to the conception and design of the study. JZ, JT and LX performed the experiments, collected the data and analyzed the data and assisted in the interpretation of the results. All authors read and approved the final manuscript.

\section{Ethics approval and consent to participate}

Not applicable.

\section{Patient consent for publication}

Not applicable.

\section{Competing interests}

The authors declare that they have no competing interests.

\section{References}

1. Moss JW and Ramji DP: Cytokines: Roles in atherosclerosis disease progression and potential therapeutic targets. Future Med Chem 8: 1317-1330, 2016.

2. Libby P, Ridker PM and Hansson GK: Progress and challenges in translating the biology of atherosclerosis. Nature 473: 317-325, 2011.

3. Glass CK and Witztum JL: Atherosclerosis. Cell 104: 503-516, 2001.

4. Horton JD, Cohen JC and Hobbs HH: Molecular biology of PCSK9: Its role in LDL metabolism. Trends Biochem Sci 32: 71-77, 2007.

5. Hedrick JA: Targeting PCSK9 for the treatment of hypercholesterolemia. Curr Opin Invest Drugs 10: 938-946, 2009.

6. Xu YJ, Zheng L, Hu YW and Wang Q: Pyroptosis and its relationship to atherosclerosis. Clin Chim Acta 476: 28-37, 2018.

7. Hoseini Z, Sepahvand F, Rashidi B, Sahebkar A, Masoudifar A and Mirzaei H: NLRP3 inflammasome: Its regulation and involvement in atherosclerosis. J Cell Physiol 233: 2116-2132, 2018.

8. Shah PK and Lecis D: Inflammation in atherosclerotic cardiovascular disease. F1000Res 8: F1000, 2019.

9. Zhaolin Z, Jiaojiao C, Peng W, Yami L, Tingting Z, Jun T, Shiyuan W, Jinyan X, Dangheng W, Zhisheng J and Zuo W: OxLDL induces vascular endothelial cell pyroptosis through miR-125a-5p/TET2 pathway. J Cell Physiol 234: 7475-7491, 2019.

10. Mottis A, Herzig S and Auwerx J: Mitocellular communication: Shaping health and disease. Science 366: 827-832, 2019.

11. Ding Z, Liu S, Wang X, Deng X, Fan Y, Sun C, Wang Y and Mehta JL: Hemodynamic shear stress via ROS modulates PCSK9 expression in human vascular endothelial and smooth muscle cells and along the mouse aorta. Antioxid Redox Signal 22: 760-771, 2015.
12. Bao HL, Liao FJ, Fang L, Zhong F, Liu W and Li JQ: Effect and mechanism of PCSK9 on lectin-like oxidized low-density lipoprotein receptor-1 mediated oxidized low-density lipoprotein uptake by THP-1 derived macrophages. Zhonghua Xin Xue Guan Bing Za Zhi 47: 367-373, 2019 (In Chinese).

13. Wu CY, Tang ZH, Jiang L, Li XF, Jiang ZS and Liu LS: PCSK9 siRNA inhibits HUVEC apoptosis induced by ox-LDL via bcl/Bax-caspase9-caspase3 pathway. Mol Cell Biochem 359: 347-358, 2012.

14. Chen JJ, Tao J, Zhang XL, Xia LZ, Zeng JF, Zhang H, Wei DH, Lv YC, Li GH and Wang Z: Inhibition of the ox-LDL-induced pyroptosis by FGF21 of human umbilical vein endothelial cells through the TET2-UQCRC1-ROS pathway. DNA Cell Biol 39: 661-670, 2020.

15. Davies PF: Hemodynamic shear stress and the endothelium in cardiovascular pathophysiology. Nat Clin Pract Cardiovase Med 6: 16-26, 2009

16. Zhang L, Yuan M, Zhang L, Wu B and Sun X: Adiponectin alleviates NLRP3-inflammasome-mediated pyroptosis of aortic endothelial cells by inhibiting FoxO4 in arteriosclerosis. Biochem Biophys Res Commun 514: 266-272, 2019.

17. Chang W, Lin J, Dong J and Li D: Pyroptosis: An inflammatory cell death implicates in atherosclerosis. Med Hypotheses 81: 484-486, 2013

18. Zhou R, Yazdi AS, Menu P and Tschopp J: A role for mitochondria in NLRP3 inflammasome activation. Nature 469: 221-225, 2011.

19. Lin XL, Xiao LL, Tang ZH, Jiang ZS and Liu MH: Role of PCSK9 in lipid metabolism and atherosclerosis. Biomed Pharmacother 104: 36-44, 2018.

20. Vozenilek AE, Vetkoetter M, Green JM, Shen X, Traylor JG, Klein RL, Orr AW and Woolard MD: Absence of nicotinamide nucleotide transhydrogenase in C57BL/6J mice exacerbates experimental atherosclerosis. J Vasc Res 55: 98-110, 2018.

21. Ruscica M, Ferri N, Corsini A and Sirtori CR: PCSK9 antagonists and inflammation. Atherosclerosis 268: 235-236, 2018.

22. Aarreberg LD, Esser-Nobis K, Driscoll C, Shuvarikov A, Roby JA and Gale M Jr: Interleukin- $1 \beta$ induces mtDNA release to activate innate immune signaling via cGAS-STING. Mol Cell 74: 801-815 e806, 2019.

23. He X, Wang L, Chen XF, Liang Q, Wang WQ, Lin AQ, Yi L, Wang Y and Gao Q: Metformin improved oxidized low-density lipoprotein-impaired mitochondrial function and increased glucose uptake involving akt-AS160 pathway in raw264.7 macrophages. Chin Med J (Engl) 132: 1713-1722, 2019.

24. Thonusin C, Apaijai N, Jaiwongkam T, Kerdphoo S, Arunsak B, Amput P, Palee S, Pratchayasakul W, Chattipakorn N and Chattipakorn SC: The comparative effects of high dose atorvastatin and proprotein convertase subtilisin/kexin type 9 inhibitor on the mitochondria of oxidative muscle fibers in obese-insulin resistant female rats. Toxicol Appl Pharmacol 382: 114741, 2019.

25. Ding Z, Pothineni NVK, Goel A, Luscher TF and Mehta JL: PCSK9 and inflammation: Role of shear stress, pro-inflammatory cytokines, and LOX-1. Cardiovasc Res 116: 908-915, 2020.

26. Ding Z, Liu S, Wang X, Mathur P, Dai Y, Theus S, Deng X, Fan Y and Mehta JL: Cross-Talk between PCSK9 and damaged mtDNA in vascular smooth muscle cells: Role in apoptosis. Antioxid Redox Signal 25: 997-1008, 2016.

27. Massaad CA and Klann E: Reactive oxygen species in the regulation of synaptic plasticity and memory. Antioxid Redox Signal 14: 2013-2054, 2011.

This work is licensed under a Creative Commons Attribution-NonCommercial-NoDerivatives 4.0 International (CC BY-NC-ND 4.0) License. 